The Astrophysical Journal, 605:840-845, 2004 April 20

(C) 2004. The American Astronomical Society. All rights reserved. Printed in U.S.A.

\title{
ON THE INFRARED, OPTICAL, AND HIGH-ENERGY EMISSION FROM THE ANOMALOUS X-RAY PULSAR 4U 0142+61
}

\author{
Ü. ERTAN ${ }^{1}$ and K. S. Cheng \\ Department of Physics, University of Hong Kong, Pokfulam Road, Hong Kong, China; unal@sabanciuniv.edu, hrspksc@hkucc.hku.hk \\ Received 2003 November 15; accepted 2004 January 5
}

\begin{abstract}
We show that the observed pulsed optical emission of the anomalous X-ray pulsar 4U $0142+61$ can be accounted for by both the magnetar outer gap models and the disk-star dynamo gap models; therefore, there is no evidence favoring only one of these models as its responsible mechanism. Nevertheless, the estimated highenergy gamma-ray spectra from these models have different power-law indices and can be tested by future observations with the Gamma-Ray Large Area Space Telescope (GLAST). Furthermore, we show by analytical estimations that the expectations of a standard disk model are in agreement with the observed unpulsed optical and infrared luminosities of the AXP $4 \mathrm{U} 0142+61$.
\end{abstract}

Subject headings: accretion, accretion disks — pulsars: individual (4U 0142+61) — stars: neutron — X-rays: bursts

\section{INTRODUCTION}

Anomalous X-ray pulsars (AXPs) are neutron stars (NSs) that are characterized by their persistent X-ray luminosities $\left(L_{\mathrm{X}} \sim 10^{34}-10^{36} \mathrm{ergs} \mathrm{s}^{-1}\right)$, which are well above that expected from rotation. Observations show no evidence for the existence of massive companions. Their periods are clustered in a remarkably narrow range $(P \sim 6-12 \mathrm{~s})$. Some of the AXPs belong to supernova remnants (SNRs), which shows that they are young objects (see Mereghetti et al. 2002 for a review of AXPs). The X-ray luminosities, periods, and blackbody temperatures of AXPs are similar to those of soft gamma-ray repeaters (SGRs; see Hurley 2000 for a review). Furthermore, recently reported SGR-like bursts from two of the AXPs (Kaspi \& Gavriil 2002; Gavriil, Kaspi, \& Woods 2002) imply that these sources probably have similar origins.

Magnetar models (Duncan \& Thompson 1992; Thompson \& Duncan 1995) are successful in explaining the fluences and the luminosities of the normal $\left(L \lesssim 10^{42} \mathrm{ergs} \mathrm{s}^{-1}\right)$ and the giant $\left(L \gtrsim 10^{44} \mathrm{ergs} \mathrm{s}^{-1}\right)$ super-Eddington bursts of SGRs. In the magnetar models, surface magnetic fields are $\gtrsim 10^{14} \mathrm{G}$, and a huge release of magnetic energy through instabilities from inside the NS is the source of these bursts. The persistent thermal X-ray emission was suggested to be due to the surface heating by magnetic field decay, while the particles accelerated in the magnetosphere by the Alfvén waves produced by the small-scale fractures on the NS surface could be the source of the nonthermal emission (Thompson \& Duncan 1996). Spin-down torque is provided by the magnetic dipole radiation. Despite the success of the magnetar model in explaining the burst energetics, it can only account for the period clustering of SGRs and AXPs in one set of decay models with special conditions (Colpi, Geppert, \& Page 2000).

On the other hand, fallback disk models, which have difficulties in explaining the short timescales of the superEddington bursts, can account for the period clustering and the persistent X-ray emission of these sources (Chatterjee, Hernquist, \& Narayan 2000; Alpar 2001; Marsden et al.

\footnotetext{
${ }^{1}$ Also at: Sabancı University, Orhanl1-Tuzla 34956, Istanbul, Turkey.
}

2001). The enhancement phase of the persistent X-ray emission following the giant flare of the SGR $1900+14$ can be explained by the postburst release of the inner disk, which has been pushed back by the burst energy (Ertan \& Alpar 2003; see also Lyubarsky, Eichler, \& Thompson 2002 for an explanation based on the crust cooling after being heated by the burst energy). Moreover, it has been shown that the young NSs with fallback disks can produce AXPs within the timescales of the ages of their associated SNRs with reasonable parameters (Chatterjee \& Hernquist 2000; Ekși \& Alpar 2003a, 2003b).

Magnetar models have no detailed predictions for the IR and optical emissions seen from the AXPs, which, in this work, is one of our main concerns regarding the disk models. Out of five observed AXPs (and two candidates), four sources were reported to have IR counterparts (Kaspi, Gavriil, \& Woods 2002; Israel et al. 2002, 2003). One of them (4U 0142+61) was also detected in the $R$ band (Hulleman, van Kerkwijk, \& Kulkarni 2000). The optical/IR luminosities of these AXPs lie above the extrapolation of the blackbody components of their X-ray spectra (Hulleman et al. 2001; Wang \& Chakrabarty 2002; Israel et al. 2003), indicating different origins for the optical/IR emission and the thermal X-rays likely to be emitted near the NS surface. The observed ratio of the total $R$-band luminosity to the X-ray luminosity is $\sim 1.4 \times 10^{-4}$ for AXP 4U 0142+61 (Hulleman et al. 2000; Hulleman 2002), claimed to be too low for the origin of the optical light to be an accretion disk. Kern \& Martin (2002) reported that the optical emission (in the $R$ band) from AXP 4U 0142+61 has a pulsed component with a pulsed fraction $h_{R} \simeq 0.27$, which is much higher than the X-ray pulsed fraction of $\sim 5 \%(1-2 \mathrm{keV})$, which strongly suggests different emission sites for the pulsed $\mathrm{X}$-ray and optical emissions. It was argued that the pulsed optical radiation is an indication that AXPs are magnetars.

In this work, we show that the pulsed optical emission can be accounted for by both the disk-star dynamo gap model (Cheng \& Ruderman 1991) and the magnetar outer gap model (Cheng \& Zhang 2001) and does not exclude one or the other ( 2 and $\S 3$ ). In $\S 4$, we show that the two models can be observationally tested through their expected different high-energy 
gamma-ray spectral characteristics. Furthermore, in $\S 5$, we show by analytical estimations that the $R$ - and $K$-band luminosities expected from a standard disk model are in agreement with the observations of the AXP 4U $0142+61$.

\section{DISK-STAR DYNAMO MODEL}

For simplicity, we consider a NS dipole magnetic field that is aligned with the spin axis. When the inner rim of a Keplerian disk rotates faster than the neutron star, a gap (accelerator) is formed around the null surface $(\boldsymbol{\Omega} \cdot \boldsymbol{B}=0)$, separating the charges in the magnetosphere. The electromotive force of the disk-star dynamo (see Cheng \& Ruderman 1991 for details; CR91 hereafter) is given by

$$
\mathcal{E}(r)=\int_{r_{0}}^{r}\left[\Omega_{\mathrm{K}}(r)-\Omega_{*}\right] B_{z}(r) \frac{r d r}{c},
$$

where $B_{z}(r)$ is the vertical component of the magnetic field through the disk, $\Omega_{\mathrm{K}}(r)$ is the local Keplerian angular velocity of the disk, $\Omega_{*}$ is the angular frequency of the NS, and $r_{0}$ is the inner radius of the disk. In a stationary gap, the electromotive force is balanced by the potential drop $V_{g}$ across the gap. Substituting $\Omega_{\mathrm{K}}(r)=\left(G M / r^{3}\right)^{1 / 2}$ into equation (1) and neglecting the effects of inflowing disk matter, we obtain

$\mathcal{E}(r)=R_{*}^{3} B_{*}\left[\frac{2}{5}\left(\frac{G M_{*}}{c^{2}}\right)^{1 / 2}\left(\frac{1}{r_{0}^{5 / 2}}-\frac{1}{r^{5 / 2}}\right)-\frac{\Omega_{*}}{c}\left(\frac{1}{r_{0}}-\frac{1}{r}\right)\right]$,

where $R_{*}, B_{*}$, and $M_{*}$ are the radius, surface magnetic field, and mass of the NS respectively. Whether the gap is closed or open depends on how close the corotation radius $r_{c}=\left(G M / \Omega_{*}^{2}\right)^{1 / 3}$ is to the inner disk radius $r_{0}$. If $r_{0}$ is less than $1.8 r_{c}$, the gap cannot close (the geometry of the open and closed gaps are given in Figs. 2 and 3 of CR91). In the limit $\Omega_{*} \ll \Omega_{\mathrm{K}}\left(r_{0}\right)$, the potential drop in equation (2) can be as high as

$\mathcal{E}(r) \sim 4 \times 10^{14} r_{0,8}^{5 / 2} B_{*, 12}\left(\frac{M}{M_{\odot}}\right)^{1 / 2} R_{*, 6}^{3}\left[1-\left(\frac{r_{0}}{r}\right)^{5 / 2}\right] \mathrm{V}$,

where we use the notation $A=A_{, i} \times 10^{i}$. This potential drop can only be reached by a static gap in which the pair production is negligible. In a more realistic stationary gap, the potential drop $V_{g}$ is limited by the flow of electrons and positrons through the gap, especially when there are copious amounts of X-ray photons traversing the gap. The X-rays are upscattered by the inverse Compton collisions with the gapaccelerated electrons and positrons. The gamma rays produced by these inverse Compton scatterings create secondary $e^{-} e^{+}$ pairs through the collisions with the X-rays. These secondary pairs emit synchrotron emission out of the gap, and we test whether this is responsible for the observed pulsed optical emission. For such a pair production-limited gap, the maximum potential drop can be estimated as

$$
V_{g}=6 \times 10^{10} L_{\mathrm{X}, 36}\left(\frac{\left\langle E_{\mathrm{X}}\right\rangle}{1 \mathrm{keV}}\right)^{-1} B_{*, 12}^{-1 / 2} R_{*, 6}^{-1} \mathrm{~V}
$$

(CR91). The energies of the primary pairs accelerated in this potential drop $V_{g}$ are limited by their collisions with the X-ray photons. On the other hand, the gap power is limited by the power of the inflowing disk matter from $r_{c}$ down to the inner disk radius $r_{0}$,

$$
\mathcal{P}_{g} \sim G M \dot{M}\left(\frac{1}{r_{0}}-\frac{1}{r_{c}}\right)
$$

The inner disk radius can be written as

$$
r_{0} \simeq \beta \frac{r_{\mathrm{A}}}{2}=2.9 \times 10^{8} \beta L_{\mathrm{X}, 36}^{-2 / 7} B_{*, 12}^{4 / 7}\left(\frac{M_{*}}{M_{\odot}}\right)^{1 / 7} R_{*, 6}^{10 / 7} \mathrm{~cm}
$$

(Ghosh \& Lamb 1979), where $r_{\mathrm{A}}$ is the Alfvén radius and $\beta \sim 1$. For the AXP $4 \mathrm{U} 0142+61, r_{c} \sim 7 \times 10^{8} \mathrm{~cm}$, and the $\mathrm{X}$-ray luminosity is $L_{\mathrm{X}}=6.5 \times 10^{35} \mathrm{ergs} \mathrm{s}^{-1}$ for a source distance of $d=3 \mathrm{kpc}$. Hulleman et al. (2000) reported $d$ to be greater than $2.7 \mathrm{kpc}$. The accretion rate is related to the X-ray luminosity through $\dot{M}=L_{\mathrm{X}} R_{*} / G M \sim 3.4 \times 10^{15} \mathrm{~g} \mathrm{~s}^{-1}$. Then equations (5) and (6) give a gap power of $\mathcal{P}_{g} \sim 1 \times 10^{33} \mathrm{ergs}$ $\mathrm{s}^{-1}$. The fraction of the gap power that is taken out of the gap by the secondary pairs depends on the pair production optical depth of the X-rays to primary gamma rays. The density of the thermal X-ray photons at $r \sim r_{0}$ can be estimated as

$$
\langle n\rangle \sim \frac{L_{\mathrm{X}}}{4 \pi\left(r_{0}\right)^{2} c\left\langle E_{\mathrm{X}}\right\rangle} \sim 10^{16} \mathrm{~cm}^{-3}
$$

where $\left\langle E_{\mathrm{X}}\right\rangle \approx 3 k T$ is the typical energy of the X-rays. For $4 \mathrm{U}$ $0142+61,3 k T \approx 1.2 \mathrm{keV}$ (Israel et al. 1999; Paul et al. 2000; Mereghetti et al. 2002; Juett et al. 2002). The optical depth for the pair production of the primary gamma rays with the soft X-rays becomes

$$
\tau \sim\langle n\rangle \sigma_{p} l \sim\left(10^{16} \mathrm{~cm}^{-3}\right)\left(10^{-25} \mathrm{~cm}^{2}\right)\left(5 \times 10^{8} \mathrm{~cm}\right) \sim 0.5
$$

where $\sigma_{p} \approx 10^{-25} \mathrm{~cm}^{2}$ is the cross-section for the pair production and $l \sim r$ is the length of the gap along the field lines ( $l$ can exceed $r$ considerably because of the azimuthal winding of the magnetic field lines across the gap). Equation (8) implies that about half of the gap power is transferred to the secondary $e^{-} e^{+}$pairs. We investigate whether the synchrotron emission from these secondary pairs just out of the gap can account for the observed optical pulsed emission from AXP 4U 0142+61.

Energy distribution of the secondary $e^{-} e^{+}$pairs can be approximated as

$$
N_{e} \sim \gamma^{-2} \ln \left(\frac{\gamma_{\max }}{\gamma}\right)
$$

(Cheng, Ho, \& Ruderman 1986), where $\gamma$ is the Lorentz factor of the secondary electrons and positrons such that $\gamma_{\min }<\gamma<$ $\gamma_{\max }$. The synchrotron energy spectrum from these secondary pairs is given by

$$
f\left(E_{\gamma}\right) \propto \int_{\gamma_{\min }}^{\gamma_{\max }} d \gamma N_{e}(\gamma) F(x),
$$

where $F(x)=x \int_{x}^{\infty} K_{5 / 3}(y) d y, K_{5 / 3}(y)$ is the modified Bessel function, $x=E_{\gamma} / E_{\mathrm{syn}}$, and $E_{\mathrm{syn}} \simeq(3 / 2) \gamma^{2} \hbar\left(e B / m_{e} c\right)$. The observed energy spectrum becomes

$$
\mathcal{F}\left(E_{\gamma}\right) \approx \frac{1}{\Delta \Omega d^{2}} f\left(E_{\gamma}\right)
$$






FIG. 1.-Photon flux spectrum of the synchrotron radiation from the magnetar outer gap model (dashed line) and the disk-star dynamo gap model (solid line). The vertical lines show the borders of the $R$ band $(1.7-2.1 \mathrm{eV})$.

where $\Delta \Omega$ is the beaming solid angle of the synchrotron emission and $d$ is the distance to the source. The total synchrotron luminosity $L_{\text {syn }}$ is found by integrating $f\left(E_{\gamma}\right)$ over all frequencies. The lower cutoff of the spectrum is determined by the minimum Lorentz factor $\gamma_{\min }$ of the secondary pairs, which we take to be 1 . The maximum Lorentz factor of the secondary pairs is $\gamma_{\max } \sim(1 \mathrm{MeV}) /\left\langle E_{x}\right\rangle \sim$ a few $\times 10^{3}$ (Cheng et al. 1986; Cheng \& Ding 1994). Since the exact position of $\gamma_{\max }$ does not affect the results significantly, we take $\gamma_{\max }=3 \times 10^{3}$. The synchrotron radiation is emitted at a radial distance $r \gtrsim r_{0}$, where the magnetic field strength reduces to about $\sim 3 \times 10^{4} \mathrm{G}$ for a dipole field with $B_{*} \sim 10^{12} \mathrm{G}$ on the surface of the NS. The resulting photon spectrum of the synchrotron emission is given in Figure 1.

Our aim is to compare the pulsed optical luminosity in the $R$ band in the model with that of the observations. The ratio of the observed optical luminosity in the $R$ band $(\sim 1.7-2.1 \mathrm{eV})$ to the X-ray luminosity was reported to be $\approx 1.4 \times 10^{-4}$ from the unabsorbed fluxes (Hulleman et al. 2000) with a pulsed fraction of the optical light of $h_{R} \sim 0.3$ (Kern \& Martin 2002). Then, the observed pulsed $R$-band luminosity is $L_{R}^{\mathrm{obs}} \sim 2.5 \times$ $10^{31}(\Delta \Omega / 4 \pi) \mathrm{ergs} \mathrm{s}^{-1}$ for $L_{\mathrm{X}} \simeq 6 \times 10^{35} \mathrm{ergs} \mathrm{s}^{-1}$, where $\Delta \Omega$ is the beaming angle of the synchrotron emission. To compare our results with the observations, we calculated the ratio of the synchrotron $R$-band luminosity to the total synchrotron luminosity and found that $L_{R} / L_{\text {syn }} \approx 1 \times 10^{-2}$. Since the pair production optical depth provided by the soft X-rays to the primary gamma rays is around $1 / 2, L_{\text {syn }} \sim \mathcal{P}_{g} / 2 \sim 5 \times 10^{32} \mathrm{ergs} \mathrm{s}^{-1}$ for the AXP $4 \mathrm{U} 0142+61$, which means $L_{R} \approx 5 \times 10^{30} \mathrm{ergs}$ $\mathrm{s}^{-1}$. For a beaming angle $\Delta \Omega \sim 2.5$, the optical pulsed luminosity estimated by the disk-star dynamo model is in agreement with the observations.

\section{MAGNETAR MODEL}

The possible gamma-ray emission properties from the outer gaps for both AXPs (with soft thermal X-ray input) and SGRs (including the effect of the hard X-ray component) have been worked out in detail by assuming dipole magnetar fields with $B_{*} \gtrsim 10^{14} \mathrm{G}$ (Cheng \& Zhang 2001; Zhang \& Cheng 2002). In the outer gap models of AXPs, the primary gamma rays are produced by the curvature emission of the accelerated primary electrons and positrons inside the gap (Cheng \& Zhang 2001), which is different from the gamma-ray production mechanism of the disk-star dynamo model. The primary gamma-ray luminosity, that is, the power provided by the magnetar outer gap, can be written as

$$
\begin{aligned}
L_{\gamma} \approx & 4.0 \times 10^{32}\left(\frac{f}{0.5}\right)^{3}\left(\frac{B_{*}}{10^{14} \mathrm{G}}\right)^{2} \\
& \times\left(\frac{P}{6 \mathrm{~s}}\right)^{-4}\left(\frac{R_{*}}{15 \mathrm{~km}}\right)^{6} \operatorname{ergs~s}^{-1}
\end{aligned}
$$

(Zhang \& Cheng 1997), where $f$ is the fraction of the volume of the outer magnetosphere occupied by the outer gap that is given by

$$
\begin{aligned}
f \approx & 0.68\left(\frac{P}{6 \mathrm{~s}}\right)^{7 / 6}\left(\frac{B}{10^{14} \mathrm{G}}\right)^{-1 / 2} \\
& \times\left[\frac{T_{S}(\theta)}{5 \times 10^{6} \mathrm{~K}}\right]^{-2 / 3}\left(\frac{R_{*}}{15 \mathrm{~km}}\right)^{-3 / 2},
\end{aligned}
$$

where $T_{S}(\theta)$ is the surface temperature of the magnetar. Its dependence on the polar angle $\theta$ with respect to the magnetic axis is weak and may be ignored. It should be noted that if $f>1$, an outer gap cannot be formed (Zhang \& Cheng 1997). For the AXP $4 \mathrm{U} 0142+61$, the reported period is $P=8.69 \mathrm{~s}$, the surface magnetic field estimated from $B=3.3 \times 10^{19}(P \dot{P})^{1 / 2}$ is around $\sim 1.4 \times 10^{14} \mathrm{G}$ (Mereghetti et al. 2002), and the corresponding fractional size of the outer gap is $f \approx 0.89$. Then the outer gap power of $4 \mathrm{U} 0142+61$ becomes $\mathcal{P}_{g} \sim 1 \times 10^{33} \mathrm{ergs} \mathrm{s}^{-1}$, which is roughly the same as the gap power of the disk-star dynamo model. In a manner similar to that of the disk-star dynamo model gap, secondary pairs, produced by the collisions between the primary gamma rays and X-ray photons originating from the NS, emit synchrotron radiation out of the gap. Unlike the diskstar dynamo gap, the outer gap of an isolated NS extends to near the light cylinder radius $r_{l}=c / \Omega_{*}$, which is $\sim 4 \times 10^{10} \mathrm{~cm}$ for 4U 0142+61. Replacing $r_{0}$ by $\sim r_{l} / 3$, where the magnetic field decreases to about $10^{2} \mathrm{G}$, and setting $l=r_{l}$ in equations (7) and (8), we obtain the pair production optical depth $\tau_{p} \sim 0.03 L_{\mathrm{X}, 36}$. For the maximum primary gamma-ray energies of approximately a few GeV (Cheng \& Zhang 2001), the maximum Lorentz factor of the secondary pairs is approximately a few $\times 10^{3}$. In the calculations, we set $\gamma_{\min }=1$ and $\gamma_{\max }=3 \times 10^{3}$. Following the same numerical calculations as that of the diskstar dynamo model, we found from the resulting spectrum (eq. [10]) that the ratio $L_{R} / L_{\text {syn }}$ is about $3 \times 10^{-2}$. The total synchrotron luminosity of the secondary pairs $L_{\text {syn }} \sim \tau_{p} \mathcal{P}_{g} \simeq 3 \times$ $10^{31} \mathrm{ergs} \mathrm{s}^{-1}$, and the corresponding pulsed $R$-band luminosity is $L_{R} \sim 3 \times 10^{-2} L_{\text {syn }} \sim 9 \times 10^{29} \mathrm{ergs} \mathrm{s}^{-1}$. With a solid angle $\Delta \Omega \sim 0.5$ of the beamed synchrotron emission, the magnetar model can also account for the observed pulsed optical luminosity of the AXP $4 \mathrm{U} 0142+61$ in the $R$ band. The expected synchrotron photon spectrum is presented in Figure 1. The weakness of the magnetar model for AXP 4U $0142+61$ is that if the surface magnetic field of this source is $\sim 1.4 \times 10^{14} \mathrm{G}$ (Mereghetti et al. 2002), then the fraction $f$ of the outer magnetosphere occupied by the outer gap (eq. [13]) exceeds unity for $R_{*}$ less than about $13.9 \mathrm{~km}$. This would mean that there is no outer gap of 4 U $0142+61$ at all (Zhang \& Cheng 1997). On the other hand, if the outer gap indeed exists in $4 \mathrm{U} 0142+61$, this implies that the equation of state (EOS) of this source cannot be soft (e.g., Shapiro \& Teukolsky 1983) with magnetar dipole fields. 
The spectral cutoff of the disk-star dynamo model lies in the $\mathrm{X}$-ray, whereas it is at about $10 \mathrm{eV}$ for the magnetar model. However, the expected pulsed X-ray luminosity of the diskstar dynamo model is not likely to be tested by observations, since it is much less than the observed pulsed X-ray emission, which is very probably emitted near the NS. In $\S 4$, we show that the two models can be distinguished through their highenergy gamma-ray spectral properties.

\section{HIGH-ENERGY EMISSION FROM THE MAGNETAR AND DISK-STAR DYNAMO MODELS}

The estimated high-energy ( $\mathrm{keV}$ to $\sim 10 \mathrm{GeV}$ ) emission spectra of the magnetar and the disk-star dynamo gap models are different, although their total high-energy luminosities are similar. The outer gaps of magnetar models, which lie close to the light cylinder, are optically thin to the inverse Compton scattering of the X-rays by the gap-accelerated pairs. These pairs emit beamed curvature radiation along the outer gap. The resulting spectra have been worked out for both AXPs and SGRs (Cheng \& Zhang 2001; Zhang \& Cheng 2002). The energy distribution of the primary $e^{-} e^{+}$pairs in the gap is given by

$$
\frac{d N}{d E_{e}} \propto\left(\frac{\gamma}{\gamma_{0}}\right)^{-16 / 3}
$$

(Zhang \& Cheng 1997), where

$$
\gamma_{0} \approx 2.8 \times 10^{7} f^{1 / 2} B_{12}^{1 / 4} P^{-1 / 4}\left(\frac{R_{*}}{10 \mathrm{~km}}\right)^{3 / 4} .
$$

The Lorentz factor $\gamma$ is given by $\gamma(x) \approx \gamma_{0} x^{-3 / 4}$ for $x_{\min } \leq$ $x \leq x_{\max }$, where $x=s / r_{l}$ and $s$ is the local radius of the curvature. For the AXP 4 U $0142+61, \gamma_{0} \sim 5 \times 10^{7}$. The value of $x_{\min }$ depends on the magnetic inclination angle $\alpha$, and it is $\sim 2 / 3$ for $\alpha=45^{\circ}$ and $\sim 2 / 5$ for $\alpha=60^{\circ}$. The value of $x_{\max }$ could be taken as $\sim 2$ (Cheng \& Zhang 2001). The primary pairs with Lorentz factors between $\gamma_{\min }$ and $\gamma_{\max }$ emit curvature radiation with typical energies $E_{\gamma} \approx(3 / 2) \hbar \gamma^{3}(c / s) \lesssim$ $1 \mathrm{GeV}$. Above the characteristic energies, the spectrum has an exponential cutoff, while $I(\nu) \propto \nu^{1 / 3}$ for $E<E_{\gamma_{\min }}$. The detailed model spectra of AXPs for different periods, magnetic fields, and inclination angles are given in Cheng \& Zhang (2001).

On the other hand, for the disk-star dynamo model, the energies of the gap-accelerated $e^{-} e^{+}$pairs are limited by the inverse Compton collisions by the X-rays. Therefore, the produced gamma-ray photons create $e^{-} e^{+}$pairs via collisions with the X-rays. The energy distribution of the pairs in the gap can be approximated by equation (9). For a power-law energy distribution $N_{e}(\gamma) \propto \gamma^{-m}$ of the pairs, the resulting intensity spectrum of the upscattered X-rays can be written as $I_{c}(\nu) \propto \nu^{-p}$ with $p=(m-1) / 2$. For the pair distribution given by equation (9), we have $m=2+\left[\ln \left(\gamma_{\max } / \gamma\right)\right]^{-1}$. The variation of the term in square brackets is small with respect to the large range of the Lorentz factors. For a variation of $\gamma_{\max } / \gamma$ from $\sim 5$ to $\sim 10^{3}, p$ changes from 0.6 to 0.8 .

We see that the expected high-energy gamma-ray spectra of the magnetar and the disk-star dynamo models have very different spectral power-law indices. The model luminosities are high enough that they can be tested by the future gamma-ray mission Gamma-Ray Large-Area Space Telescope (GLAST) (Cheng \& Zhang 2001).

\section{UNPULSED OPTICAL/IR EMISSION}

Since the unpulsed component of the optical/IR emission is expected to be isotropic, there is no beaming correction, and therefore $\left(L_{R} / L_{\mathrm{X}}\right)_{\mathrm{obs}} \sim 10^{-4}$ for the unpulsed emission of the AXP 4U 0142+61. The reported IR and optical magnitudes are $K \approx 19.4 \mathrm{mag}$ and $R \approx 25 \mathrm{mag}$ (Hulleman et al. 2000; Hulleman 2002). The estimated interstellar extinction in the $R$ band is $A_{R}=4.4 \mathrm{mag}$; therefore, it is expected that $A_{K} \simeq 0.5$ in the IR band. We estimate that the ratio of the observed $K$-band flux to that of the $R$ band $\left(L_{K} / L_{R}\right)_{\text {obs }}$ is $\sim 10^{-1}$. We compare these observations with the expectations of a standard accretion disk model.

We take $\dot{M}_{*}=\eta \dot{M}_{\text {in }}$, where $\eta$ is the fraction of the disk mass flow rate which is accreted onto the NS, and assume that the mass flow through the inner disk regions emitting in the $K$ and $R$ bands can be represented by a steady state mass flow. The local dissipation rate $D(r)$ of the disk at the radial distance $r$ in steady state is given by

$$
D(r) \simeq \frac{3}{8 \pi} \dot{M} \frac{G M}{r^{3}} .
$$

For $L_{\mathrm{X}} \sim 10^{36}$ ergs $\mathrm{s}^{-1}$, the accretion rate onto the NS becomes $\dot{M}_{*} \sim 5 \times 10^{15} \mathrm{~g} \mathrm{~s}^{-1} \sim \eta \dot{M}_{\text {in }}$. We obtain

$$
D(r) \approx 1.2 \times 10^{14} r_{9}^{-3} \eta^{-1} \mathrm{ergs} \mathrm{s}^{-1} \mathrm{~cm}^{-2},
$$

where $r_{9}$ is the radial distance in units of $10^{9} \mathrm{~cm}$. The corresponding local effective temperature of the disk becomes

$$
T_{\mathrm{eff}}(r)=\left[\frac{D(r)}{\sigma}\right]^{1 / 4} \simeq 3.8 \times 10^{4} r_{9}^{-3 / 4} \eta^{-1 / 4},
$$

where $\sigma$ is the Stefan-Boltzmann constant. The central wavelength of the $R$ band is $\lambda_{R} \simeq 138 \mathrm{~nm}$. The effective temperature that gives the maximum of its blackbody emission in the $R$ band is $k T_{\text {eff }} \sim 1 \mathrm{eV}$. Equating $k T_{\text {eff }}=1 \mathrm{eV}$ in equation (17), we find $r\left(k T_{\text {eff }} \sim 1 \mathrm{eV}\right) \sim 4.8 \times 10^{9} \eta^{-1 / 3} \mathrm{~cm}$. Then

$$
\frac{L_{R}}{L_{\mathrm{X}}} \simeq\left[\frac{R_{*}}{2 r\left(k T_{\mathrm{eff}}=1 \mathrm{eV}\right)}\right] \simeq 1 \times 10^{-4} \eta^{-2 / 3},
$$

which is in agreement with the observed ratio reported by Hulleman et al. (2000) for the values of $\eta$ near unity. To estimate the IR flux from the disk, we equate $k T_{\text {eff }} \simeq 0.1 \mathrm{eV}$ in equation (17) and find $r\left(k T_{\text {eff }} \simeq 0.1 \mathrm{eV}\right) \simeq 1 \times 10^{2} r_{9} \simeq$ $10^{11} \eta^{-1 / 3} \mathrm{~cm}$. Equation (17) gives $\left(L_{K} / L_{\mathrm{X}}\right) \sim 10^{-5} \eta^{-2 / 3}$; that is, $L_{K} / L_{R} \sim 10^{-1}$ (independent of $\eta$ ), which is also consistent with the observations of the AXP 4 U $0142+61$. On the other hand, shorter wavelength optical and UV emission, which is expected from the innermost regions of the disk, could be effectively absorbed due to increasing interstellar absorption with decreasing wavelength along these energies.

Our estimates remain consistent with the observations as long as $\eta$ is greater than about $\sim 30 \%$. Ekşi \& Alpar (2003a) showed that fallback disks can produce the AXP period and period derivatives in timescales consistent with their SNR ages by using dipole magnetic fields with $B_{*} \sim 10^{12}-10^{13} \mathrm{G}$. They estimated that $\eta$ could be as low as 0.01 for some AXPs 
and pointed out that $\eta$ could be higher if the mass loss from the disk due to the propeller effect during the lifetime of the AXPs and SGRs was taken into account. From the disk model parameters of AXP 0142+61 (Ekși \& Alpar 2003b), we can estimate its present disk mass flow rate as $\sim 3 \times 10^{16} \mathrm{~g} \mathrm{~s}^{-1}$, that is, $\eta \sim 15 \%$, which is consistent with our rough estimate within a factor of $\sim 2-3$. More detailed numerical calculations of the optical/IR emission from the AXPs and SGRs will be presented in a separate work.

In present disk models of AXPs, IR and optical luminosities are assumed to be dominated by the irradiation of the disk by the central X-rays (Perna, Hernquist, \& Narayan 2000; Perna \& Hernquist 2000). For the observed IR emission from the AXP 1RXS J1708-40, these X-ray-irradiated disk models give unreasonably large inner disk radii $\left(>10^{11} \mathrm{~cm}\right)$ and restrict the radial extension of the disk $\left(\Delta R \sim 5 \times 10^{11}-10^{12} \mathrm{~cm}\right.$; Israel et al. 2003). Optical/IR emission from the low-mass $\mathrm{X}$-ray binary disks has long been known to be dominated by $\mathrm{X}$-ray irradiation, and this could be the motivation to include the X-ray irradiation in the AXP disk models. However, X-ray irradiation could be inefficient for the AXP disks for the following reason. The observationally supported irradiation of the outer disk of low-mass X-ray binaries (LMXBs) has to be indirect, which is very probably due to a central extended hot corona (see, e.g., Dubus et al. 1999) because of the selfscreening of the disk. A central extended hot corona can be fed by the thermally unstable matter from the inner disk where the thermal instabilities are most effective (Shaviv \& Wehrse 1986). However, the innermost disk regions of AXPs (and SGRs) are cut by the dipole magnetic fields of their neutron stars, which are orders of magnitude higher than the magnetic fields of the NSs in LMXBs. Therefore, the X-ray irradiation that prevails at the LMXB disks is unlikely to be effective for the AXP and SGR disks, although it is not impossible. Therefore, in our calculations we ignore the X-ray irradiation. The observed IR/optical luminosity of AXPs does not restrict the outer disk radius in our explanation. That is, the outer disk expands freely in the low-viscosity state and needs not to be passive (no viscosity) as proposed by Menou, Perna, \& Hernquist (2001). On the other hand, we expect that the inner disk radius is around the Alfvén radius $\left(\sim 5 \times 10^{8} \mathrm{~cm}\right)$, which is about an order of magnitude less than the estimated radial distance at which most of the optical $R$-band luminosity is emitted $\left(\sim 5 \times 10^{9} \mathrm{~cm}\right)$. The inner rim of the disk can be irradiated by the $\mathrm{X}$-rays, but the resulting emission is expected to be in the UV band, which is easily absorbed by the interstellar medium. It is difficult to estimate the detailed emission spectrum from the innermost disk because of the uncertainties in the physics of the disk-magnetosphere interaction. Part of the inner disk matter that is outflowing because of the propeller effect may also modify the emission from the underlying innermost disk from which higher energy optical photons are emitted.

\section{CONCLUSIONS AND DISCUSSION}

We have shown that: (1) the observed optical pulsed emission from the AXP 4 U $0142+61$ can be explained by means of both the magnetar outer gap model and the disk-star dynamo gap model and therefore does not eliminate either of the models as its responsible mechanism, (2) the two models, which do not provide an observational test through their different synchrotron X-ray luminosity because of the dominating X-ray luminosity from the NS, can be tested observationally via their estimated high-energy gamma-ray spectra by GLAST, and (3) the IR and optical emission expected from a standard accretion disk model are in agreement with the reported unpulsed IR and optical luminosity of the AXP 4U 0142+61 for a ratio of the accretion rate onto the NS to the disk mass flow rate of $\eta \gtrsim 30 \%$.

Our estimation for $\eta$ is roughly consistent with that obtained from the fallback disk model of AXP 4U 0142+61 (Ekşi \& Alpar 2003b). The IR-to-X-ray luminosity ratio of the AXP 1E $2259+58$ is similar to that of $4 \mathrm{U} 0142+61$ (Hulleman 2002) and can be explained by similar arguments. The reported $L_{R} / L_{\mathrm{X}}$ ratio of AXP $1 \mathrm{RXS} \mathrm{J1708-40} \mathrm{is} \sim 2 \times$ $10^{-3}$ (Israel et al. 2003), which is about 10 times higher than that of $4 \mathrm{U} 0142+61$ and might indicate relatively more efficient mass outflow due to the propeller effect in this system with $\eta \sim 1 / 30$. Detailed numerical disk calculations to estimate the optical/IR luminosities of AXPs and SGRs and comparisons with their period and period evolution models will be presented in a future work.

We thank Ali Alpar and Yavuz Ekși for discussions and comments on the manuscript. Ü. E. acknowledges support from the High Energy Astrophysics Research Group TBAG-Ç-4 of TÜBITAK and from the Astrophysics and Space Forum at Sabancı University. Part of this work was supported by an RGC grant of Hong Kong Government.
Alpar, M. A. 2001, ApJ, 554, 1245

Chatterjee, P., \& Hernquist, L. 2000, ApJ, 543, 368

Chatterjee, P., Hernquist, L., \& Narayan, R. 2000, ApJ, 534, 373

Cheng, K. S., \& Ding, W. K. Y. 1994, ApJ, 431, 724

Cheng, K. S., Ho, C., \& Ruderman, M. 1986, ApJ, 300, 522

Cheng, K. S., \& Ruderman, M. 1991, ApJ, 373, 187 (CR91)

Cheng, K. S., \& Zhang, L. 2001, ApJ, 562, 918

Colpi, M., Geppert, U., \& Page, D. 2000, ApJ, 529, L29

Dubus, G., Lasota, J.-P., Hameury, J.-M., \& Charles, P. 1999, MNRAS, 303, 139

Duncan, R. C., \& Thompson, C. 1992, ApJ, 392, L9

Ekși, K. Y., \& Alpar, M. A. 2003a, ApJ, 599, 450 2003b, in Proc. II BeppoSAX Meeting: The Restless High-Energy

Universe, ed. E. P. J. van den Heuvel, J. J. M. in 't Zand, \& R. A. M. J.

Wijers (Amsterdam: Elsevier), in press (astro-ph/0309029)

Ertan, Ü., \& Alpar, M. A. 2003, ApJ, 593, L93

Gavriil, F. P., Kaspi, V. M., \& Woods, P. M. 2002, Nature, 419, 142

Ghosh, P., \& Lamb, F. 1979, ApJ, 234, 296
REFERENCES

Hulleman, F. 2002, in ASP Conf. Ser. 271, Neutron Stars in Supernova Remnants, ed. P. O. Slane \& B. M. Gaensler (San Francisco: ASP), 282

Hulleman, F., Tennant, A. F., van Kerkwijk, M. H., Kulkarni, S. R., Kouveliotou, C., \& Patel, S. K. 2001, ApJ, 563, L49

Hulleman, F., van Kerkwijk, M. H., \& Kulkarni, S. R. 2000, Nature, 408, 689 Hurley, K. 2000, in AIP Conf. Proc. 526, Gamma-Ray Bursts: 5th Huntsville Symp., ed. R. M. Kippen, R. S. Mallozzi, \& G. J. Fishman (New York: AIP), 763

Israel, G. L., Covino, S., Stella, L., Campana, S., Haberl, F., \& Mereghetti, S. 1999, ApJ, 518, L107

Israel, G. L., et al. 2002, ApJ, 580, L143 2003, ApJ, 589, L93

Juett, A. M., Marshall, H. L., Chakrabarty, D., \& Schulz, N. S. 2002, ApJ, 568, L31

Kaspi, V. M., \& Gavriil, F. P. 2002, IAU Circ. 7924, 3

Kaspi, V. M., Gavriil, F. P., \& Woods, P. M. 2002, IAU Circ. 7926, 1

Kern, B., \& Martin, C. 2002, Nature, 417, 527

Lyubarsky, Y., Eichler, D., \& Thompson, C. 2002, ApJ, 580, L69 
Marsden, D., Lingenfelter, R. E., Rothschild, R. E., \& Higdon, J. C. 2001, ApJ, 550,397

Menou, K., Perna, R., \& Hernquist, L. 2001, ApJ, 559, 1032

Mereghetti, S., Chiarlone, L., Israel, G. L., \& Stella, L. 2002, in Proc. 270th WE-Heraus Seminar on Neutron Stars, Pulsars and Supernova Remnants, ed. W. Becker, H. Lesch, \& J. Trümper (MPE Rep. 278; Garching: MPE), 29

Paul, B., Kawasaki, T., Dotani, T., \& Nagasake, F. 2000, ApJ, 537, 319

Perna, R., \& Hernquist, L. 2000, ApJ, 544, L57

Perna, R., Hernquist, L., \& Narayan, R. 2000, ApJ, 541, 344

Shapiro, S. L., \& Teukolsky, S. A. 1983, Black Holes, White Dwarfs, and Neutron Stars: The Physics of Compact Objects (New York: Wiley)
Shaviv, G., \& Wehrse, R. 1986, A\&A, 159, L5

Thompson, C., \& Duncan, R. C. 1995, MNRAS, 275, 255

1996, ApJ, 473, 322

Wang, Z., \& Chakrabarty, D. 2002, in ASP Conf. Ser. 271, Neutron Stars in Supernova Remnants, ed. P. O. Slane \& B. M. Gaensler (San Francisco: ASP), 297

Zhang, L., \& Cheng, K. S. 1997, ApJ, 487, 370

-. 2002, ApJ, 579, 716 